Relations industrielles

Industrial Relations

\title{
Working for McDonald's in Europe: The Unequal Struggle? par \\ Tony ROYLE, London : Routledge, 2000, 248 p., ISBN \\ 0-415-20787-8.
}

\section{Gilles Marcoux}

Volume 58, numéro 4, automne 2003

URI : https://id.erudit.org/iderudit/007832ar

DOI : https://doi.org/10.7202/007832ar

Aller au sommaire du numéro

Éditeur(s)

Département des relations industrielles de l'Université Laval

ISSN

0034-379X (imprimé)

1703-8138 (numérique)

Découvrir la revue

Citer ce compte rendu

Marcoux, G. (2003). Compte rendu de [Working for McDonald's in Europe:

The Unequal Struggle? par Tony ROYLE, London : Routledge, 2000, 248 p.,

ISBN 0-415-20787-8.] Relations industrielles / Industrial Relations, 58(4),

724-726. https://doi.org/10.7202/007832ar

Tous droits réservés (C Département des relations industrielles de l'Université Laval, 2003
Ce document est protégé par la loi sur le droit d'auteur. L'utilisation des services d'Érudit (y compris la reproduction) est assujettie à sa politique d'utilisation que vous pouvez consulter en ligne.

https://apropos.erudit.org/fr/usagers/politique-dutilisation/ 
development of workers' organizations in Indonesia. But it is only an introduction, and I hope that others will provide the focus that La Botz suggested he would provide before he decided to turn to Russia in 1905.

KIM SCIPES

University of Illinois at Chicago

\section{Working for McDonald's in Europe: The Unequal Struggle?}

par Tony RoYLE, London : Routledge, 2000, 248 p., ISBN 0-415-20787-8.

Eu égard aux structures de régulation nationale ou supranationale des régions où elles exercent leurs activités, les multinationales adaptent-elles ou imposentelles leurs politiques de gestion en matière de relations du travail ? Les droits des travailleurs sont-ils ou non protégés par ces mesures de régulation? Voilà les questions majeures qui guident cet ouvrage de Tony Royle dont la réflexion s'inspire des politiques de gestion en matière d'emploi qu' applique en Europe la multinationale McDonald's. À partir de ces interrogations, l'auteur en arrive au constat suivant : le type de conflit émanant des divers systèmes nationaux de régulation entre le mouvement syndical et l'entreprise constitue le résultat inéluctable d'une lutte entre deux groupes d'acteurs d'inégales forces, compte tenu de la faveur dont bénéficie actuellement l'idéologie néolibérale dans le contexte de mondialisation économique. En ce sens, une société aussi puissante que McDonald's parvient bien souvent à escamoter ces droits des travailleurs et à garder intact ses principes de gestion étroitement liés au libéralisme économique (comme son anti-syndicalisme), et ce, même sur le continent européen souvent perçu comme le dernier bastion occidental de la protection collective des droits démocratiques des travailleurs.

D'une durée totale d'environ six ans, le travail de recherche de Royle débuta par un examen des pratiques en matière d'emploi de McDonald's en Allemagne et au Royaume-Uni pour ensuite s'étendre à onze autres pays européens. Le travail empirique de cette étude s'inspira de diverses techniques de cueillettes de données telles que le dépouillement de statistiques officielles, des périodes d'observation participante et des entrevues réalisées avec la haute direction de la firme, les gérants de restaurants, les représentants syndicaux et les travailleurs.

Outre les parties d'introduction et de conclusion, l'ouvrage de Royle se divise en sept chapitres principaux desquels émanent cinq thématiques : les grandes lignes de l'historique de McDonald's, son expansion au-delà des frontières ainsi que sa structure et son organisation sur le marché européen (chap. 2) ; une description de son système de franchisés, de son organisation du travail et de sa culture d'entreprise (chap. 3 et 4 ) ; un examen des problèmes liés à la syndicalisation et à l'application de conventions collectives dans ses restaurants européens (chap. 5) ; une description des divers modèles législatifs nationaux européens concernant les droits de représentation et de participation des travailleurs sur leurs lieux de travail ainsi qu'une analyse des impacts de ces mécanismes légaux sur les conditions de travail des employés chez McDonald's (chap. 6 et 7); une analyse d'une portion de la législation supranationale de l'Union européenne touchant les multinationales, soit celle portant sur les comités d'entreprise européens (chap. 8).

De ces thématiques se dégagent trois idées centrales. D'abord, l'analyse portant sur le système de franchisés, l'organisation du travail et la culture d'entreprise de McDonald's apprend au lecteur que les franchises constituent des entités indépendantes de la maison-mère légalement mais dépendantes économiquement. Cette double réalité confère à la firme des possibilités accrues de 
surveillance et de contrôle à distance tout en la déresponsabilisant si certaines pratiques des franchisés heurtent les droits des travailleurs. De plus, en vertu du caractère spécifique du bassin de main-d'œuvre à l'intérieur duquel la firme recrute plusieurs de ses employés, McDonald's parvient efficacement à camper ses pratiques de gestion sur des principes tels que le recruited acquiescence (p. 82-84) et le management of subjectivity (p. 63-67), deux concepts facilitant le maintien uniforme de son système de gestion en matière de relations d'emploi.

Ensuite, l'examen des problèmes liés à la syndicalisation et à l'application des modèles législatifs nationaux de régulation révèle que les droits de participation et de représentation syndicale des travailleurs dans les restaurants McDonald's européens sont bien souvent escamotés par la firme. En fait, les importantes failles juridiques contenues dans ces mécanismes de régulation (les comités d'entreprise) permettent à la firme de développer des stratégies de contournement des droits des travailleurs, la diffusion sans encombre du modèle de participation propre à McDonald's (la McParticipation) pouvant alors se réaliser.

Enfin, l'analyse de Royle porte sur une partie de la législation de l'Union européenne affectant les multinationales, soit celle concernant les comités d'entreprise européens. Cet accord législatif supranational requiert la mise en place, pour chaque multinationale œuvrant en Europe, d'un comité mixte employeuremployés chargé de développer une base d'échanges et de discussions sur divers enjeux touchant la firme et les employés. Or, cet accord comporte encore une fois des failles juridiques importantes : l'entente est établie sur l'idée d'adhésion volontaire des multinationales, elle est dépourvue de sanctions envers les firmes ne se conformant pas à ses objectifs d'application et les franchisés n'ont pas à se soumettre à cet accord. Alors, les comités prennent la forme d'un centre de discussion servant à promouvoir l'agenda managérial (p. 206), les failles de l'accord permettant entre autres à la firme de nommer sur ces comités des gérants salariés comme représentants d'employés.

Bref, l'ouvrage de Royle demeure une étude à recommander à tous ceux qui s'intéressent à la mondialisation et à ses impacts possibles sur l'interaction entre ce phénomène et les mécanismes de régulation nationale ou supranationale en matière d'emploi. L'étude est très détaillée et son analyse s'appuie solidement sur des données recueillies avec minutie. Cependant, nous formulerions à son sujet un commentaire qui n'altère en rien sa qualité remarquable.

À la fin du chapitre 8, l'auteur soutient que le temps est venu soit de resserrer les principes juridiques de cet accord, soit de renforcer les modèles de régulation nationaux (p. 195). Or, comment y arriver ? Un accord législatif supranational tel celui concernant les comités d'entreprise européens est-il impulsé par une réelle volonté politique de lui attribuer un pouvoir de sanctions juridiques à l'égard des firmes ne se conformant pas à ses objectifs d'application? L'adhésion volontaire des multinationales à cet accord est-elle compatible à l'imposition de telles sanctions ? Serait-il avantageux de s'inspirer des modèles de régulation nationaux qui semblent disposer d'un certain pouvoir de sanction? En contexte de mondialisation économique, une telle décision risque-t-elle d'amener les multinationales à se tourner vers des territoires nationaux moins contraignants ? En outre, comment améliorer l'articulation entre les visées de l'accord supranational et l'action syndicale internationale européenne?

Il s'agit là de questions fort pertinentes si l'on cherche à définir d'éventuelles solutions pour pallier aux faiblesses juridiques de l'accord supranational 
étudié. À notre avis, il aurait été certainement intéressant que l'auteur recueille le point de vue de quelques représentants politiques de l'Union européenne pour à la fois aller au-delà du constat d'inefficacité de cet accord, offrir des pistes de réflexion à l'égard de futurs travaux de recherche s'intéressant à la portée de ce genre d'entente supranationale, et ainsi déterminer si celle-ci peut être vouée à un avenir se rapprochant plus du pragmatisme que du simple symbolisme pour les travailleurs.

Gilles Marcoux

Université Laval 